Gut and Liver, Vol. 11, No. 4, July 2017, pp. 455-463

\title{
Inflammatory Bowel Disease: Updates on Molecular Targets for Biologics
}

\author{
Konstantinos H. Katsanos ${ }^{1}$ and Konstantinos A. Papadakis ${ }^{2}$ \\ ${ }^{1}$ Division of Gastroenterology, Department of Internal Medicine, University of Ioannina School of Health Sciences, Ioannina, Greece, and \\ ${ }^{2}$ Division of Gastroenterology and Hepatology, Mayo Clinic, Rochester, MN, USA
}

Therapy for inflammatory bowel disease (IBD) has changed, with several new agents being evaluated. The era of anti-tumor necrosis factor (anti-TNF) antibody therapy saw remarkable progress in IBD therapy. Some patients, however, do not respond to anti-TNF treatment, or their response decreases over time. This phenomenon highlights the need to identify new molecular targets for therapy in IBD. The targets of new therapeutic molecules in IBD must aim to restore immune dysregulation by the inhibition of proinflammatory cytokines (TNF- $\alpha$, interleukin [IL]-6, IL-13, IL-17, IL-18, and IL-21) and augmentation of the effect of anti-inflammatory cytokines (IL-10, IL-11, and transforming growth factor $\beta$ ) and to pursue new anti-inflammatory targets, such as regulatory T-cell therapy, Smad7 antisense, Janus-activated kinase inhibition, Toll-like receptor stimulation, leukocyte adhesion, and blockade of T-cell homing via integrins and mucosal addressin cellular adhesion molecule-1. In addition, potential molecular targets could restore mucosal barrier function and stimulate mucosal healing. Despite these potential targets, the value and clinical significance of most new molecules remain unclear, and clinical efficacy and safety must be better defined before their implementation in clinical practice. This article aims to review the promising and emerging molecular targets that could be clinically meaningful for novel therapeutic approaches. (Gut Liver 2017;11:455-463)

Key Words: Crohn disease; Inflammatory bowel disease; Molecular targets; Therapy targets; Colitis, ulcerative

\section{INTRODUCTION}

Inflammatory bowel disease (IBD), specifically Crohn's disease (CD) and ulcerative colitis (UC), are autoimmune diseases whose incidence and prevalence are increasing worldwide. The cause of IBD is still unknown but is generally considered to be multi- factorial. Genetic factors are hypothesized to have a substantial role, in parallel with environmental, infectious, and immunologic factors. During the past few years, substantial progress has been made in understanding the pathogenetic mechanisms of IBD. Recent studies have examined the concept that IBD could result from dysregulation of the intestinal barrier and a pathologic activation of the intestinal immune response toward several bacterial or viral antigens. ${ }^{1,2}$ In the past few decades, the substantial progress made in understanding the pathophysiology of IBD has been translated into newer, more effective therapies-biologic and molecular therapies-that have decreased the occurrence of flares, led to remission in more patients, and improved patients' quality of life (Table 1).

In general, our knowledge of the immune system and its dysregulation in IBD is derived from mouse models of colitis and from human studies involving clinical and laboratory experiments. Emerging data support a major role for both innate and adaptive immunity in the onset and the perpetuation of chronic intestinal inflammation (Fig. 1). Of interest, meta-analyses of genomewide association studies in IBD have demonstrated several susceptibility genes involved in innate mucosal defense and antigen presentation. In addition DLG5, MDR1, NOD2 and PPAR- $\gamma$ genes are also considered to be very important players in this process. ${ }^{3}$

This progress has led to the identification of important molecules of the immune system that could represent promising targets for new molecular therapies. For example, molecules that could represent ideal targets for biologic therapies include several interleukins (ILs), tumor necrosis factor (TNF), nuclear factor- $\mathrm{\kappa} \mathrm{B}$, and antisense oligonucleotides. ${ }^{4.5}$ The aim of this review is to provide an overview of the promising and emerging molecular targets that could be clinically meaningful for novel therapeutic approaches.

Correspondence to: Konstantinos A. Papadakis

Division of Gastroenterology and Hepatology, Mayo Clinic, 200 First Street SW, Rochester, MN 55905, USA

Tel: +1-507-266-0873, Fax: +1-507-284-0538, E-mail: Papadakis.konstantinos@mayo.edu

Received on June 23, 2016. Revised on August 3, 2016. Accepted on August 3, 2016. Published online May 11, 2017

pISSN 1976-2283 eISSN 2005-1212 https://doi.org/10.5009/gnl16308

@. This is an Open Access article distributed under the terms of the Creative Commons Attribution Non-Commercial License (http://creativecommons.org/licenses/by-nc/4.0) which permits unrestricted non-commercial use, distribution, and reproduction in any medium, provided the original work is properly cited. 
Table 1. Potential Molecular Targets for Biologic Therapies in Patients with Inflammatory Bowel Disease

\begin{tabular}{|c|c|}
\hline Dysregulated molecular mechanism(s) in IBD & Potential molecular target(s) \\
\hline \multicolumn{2}{|l|}{ A. Intestinal epithelial barrier dysfunction } \\
\hline Apoptosis & Apoptotic molecules (e.g., caspase-8) \\
\hline Translocation of antigens/microbes & Toll-like receptors (e.g., TLR-4) \\
\hline Antigen-presenting cells & Macrophages, dendritic cells \\
\hline Paneth cells & Defensins \\
\hline \multicolumn{2}{|l|}{ B. Acute inflammation } \\
\hline Failure of regulatory cells & Regulatory T cells \\
\hline \multirow[t]{4}{*}{ Activation of proinflammatory mediators } & T effector cells (Th1, Th2, Th17) \\
\hline & B cells \\
\hline & Dendritic cells \\
\hline & Macrophages (TGF $\beta$, TNF- $\alpha$, IFN- $\gamma$, cytokines [IL-6, IL-9, IL-12, IL-23]) \\
\hline \multirow[t]{2}{*}{ Signaling pathways } & Smad7 \\
\hline & JAK inhibitors (e.g., tofacitinib) \\
\hline \multirow[t]{2}{*}{ Trafficking pathways } & Adhesion molecules (e.g., MAdCAM-1) \\
\hline & Anti-integrins (e.g., anti- $\alpha 4 \beta 7$ ) \\
\hline \multicolumn{2}{|l|}{ C. Perpetuation of chronic inflammation } \\
\hline Innate intestinal immunity mechanisms & Genes involved in innate mucosal defense and antigen presentation (NOD2, MDR1, PPAR- $\gamma$ ) \\
\hline \multirow[t]{3}{*}{ Adaptive intestinal immunity mechanisms } & Regulatory T cells \\
\hline & T effector cells (Th) \\
\hline & B cells \\
\hline Oxidative stress balance & Redox-sensitive signaling pathways and proinflammatory transcription molecules \\
\hline \multirow[t]{2}{*}{ D. Mucosal healing, tissue destruction } & Dendritic cells, adipocytes \\
\hline & Fibroblasts, myofibroblasts \\
\hline
\end{tabular}

IBD, inflammatory bowel disease; Th, T helper; TGF $\beta$, transforming growth factor $\beta$; TNF- $\alpha$, tumor necrosis factor $\alpha$; IFN, interferon; IL, interleukin; JAK, Janus-activated kinase; MAdCAM-1, mucosal addressin cellular adhesion molecule-1.

\section{TARGETING INTESTINAL EPITHELIAL BARRIER DYSFUNCTION}

\section{Epithelial barrier}

The epithelial cells comprise enterocytes, goblet cells, neuroendocrine cells, Paneth cells, and microfold cells (or M cells). ${ }^{6}$ The epithelial cells are sealed with intercellular tight junctions that serve a barrier function and regulate the trafficking of macromolecules between the luminal environment and the host. ${ }^{7}$ Despite this barrier, gut bacteria and luminal antigens do enter the subepithelial lamina, primarily through the specialized epithelium that overlies the organized lymphoid tissue of the gastrointestinal tract. Furthermore, polymorphisms in several IBD-related genes seem to primarily affect intestinal permeability and may result in an uncontrolled and potentially harmful exposure of the mucosal immune system to various microbial, viral, or other antigens. ${ }^{8-11}$

\section{Apoptosis regulation}

The control of cell death in the intestinal epithelium is delicately balanced. In fact, excessive cell death may cause epi- thelial barrier dysfunction and uncontrolled translocation of several luminal antigens into the intestinal wall. In patients with IBD, susceptibility to chronic inflammation of the gut can be the result of such epithelial barrier dysfunction. By contrast, various defects in cell life-death turnover may result in an aberrant accumulation of epithelial cells and may cause bowel mucosa dysplasia or even cancer. For example, many studies on necroptosis have demonstrated that caspase inhibition may directly result in intestinal barrier dysfunction and subsequent gut inflammation. $^{12}$

Caspases are proteases that regulate cell differentiation, homoeostasis, and removal of damaged cells from the intestine, as well as other organs of the body. Their activity is regulated to allow rapid activation and optimal response to any type of stress. In recent years, caspase dysregulation has been related to several diseases, including IBD. Of note, caspases-1, -4, -5, and -12 are activated during innate immune responses and actively contribute to the formation of the inflammasome. Caspase- 8 controls necroptosis of Paneth cells and appears to be involved in chronic inflammation of the intestinal epithelium. In fact, caspase-8 seems to represent a crucial player in cell survival, 


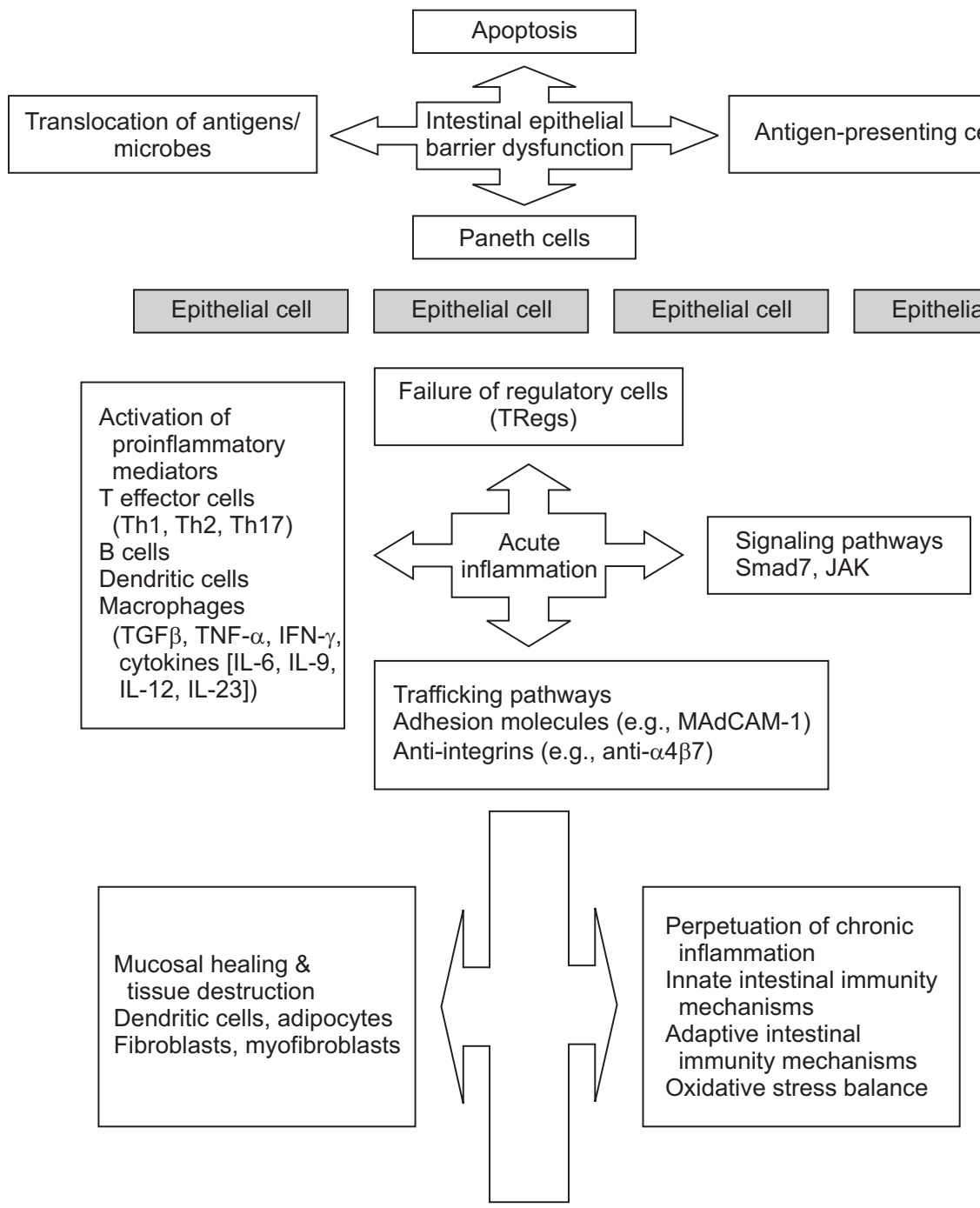

Fig. 1. Summary and schematic illustration of the series of events involved in the pathogenesis of inflammatory bowel disease in the intestine.

Th, T helper; TGF $\beta$, transforming growth factor $\beta$; TNF- $\alpha$, tumor necrosis factor $\alpha$; IFN, interferon; IL, interleukin; TRegs, regulatory T cells; JAK, Janus-activated kinase; MAdCAM-1, mucosal addressin cellular adhesion molecule- 1 .

death, and regulation of the type of cell death. Caspase-8 dysregulation has been shown to result in epithelial cell death and intestinal inflammation. Regulators of caspase- 8 could be hypothesized to prevent cell death and apoptosis dysregulation in patients with IBD. Further research on caspase regulation may improve our ability to control the function of caspases in the intestinal epithelium and may indicate new types of therapies for IBD. ${ }^{13}$

Another important regulator of bowel epithelium homeostasis is the caudal-related homeobox transcription factor 2 (CDX2), which regulates various processes such as proliferation, adhesion, and migration of intestinal cells. Some data also show involvement of CDX2 in proinflammatory pathways and in regulation of genes directly related to intestinal inflammation. Of importance, CDX2 expression was shown to be decreased in patients with UC and was inversely correlated with TNF- $\alpha$ levels. ${ }^{14}$

\section{Translocation of antigens/microbes: receptors and transporters}

\section{1) Toll-like receptors}

The cells of the innate immune system, in contrast to the cells of the adaptive immune system, have a much more rapid and less specific response to invading microorganisms or toxic macromolecules. This response is mediated by pathogen-recognition receptors, which are membrane bound, such as the Toll-like receptors (TLRs). These receptors recognize predefined molecular structures, and this recognition leads to a rapid response. ${ }^{15}$

The characterization of TLRs has helped our understanding of the initiating events of the inflammatory response in IBD. The role of TLRs as potential therapeutic targets in patients with IBD, however, is yet to be investigated. TLRs are expressed on various immune and epithelial cells and regulate the immune response to external antigens through recognition of pathogenassociated molecular patterns. In addition, TLRs seem to also interact with various endogenous human ligands regulating dif- 
ferent cell processes. According to previous data, ${ }^{16}$ TLRs could represent another potential molecular target for IBD therapy. In fact, experimental drugs modulating TLR signaling have demonstrated remarkable efficacy profiles in controlling intestinal inflammation.

The potential molecular targeting of TLRs must to be used with caution and in such way not to oversuppress the innate immune response or dysregulate other important signaling pathways. The great challenge with using TLRs as therapeutic targets is to find the optimum balance between immune system suppression and effective immune response. In the coming years, the production of TLR-based molecular therapies for IBD is expected to respect this immune system balance to favor the IBD healing process. ${ }^{17}$

\section{2) Organic cation transporters}

The organic cation transporter (OCT) family is known to have various interactions with several exogenous antigens. OCTN1 uses acetylcholine as its physiologic substrate, and OCTN2 regulates carnitine homeostasis. Of note, both OCTN1 and OCTN2 have been linked to various autoimmune diseases, including IBD, and thus represent useful pharmacologic targets. Their potential in effective IBD therapeutics is yet to be elucidated. ${ }^{18}$

\section{Antigen-presenting cells}

\section{1) Macrophages}

During pathogen invasion and inflammation, intestinal macrophages recruited from blood monocytes rapidly convert to a proinflammatory phenotype. Once activated, the macrophages express receptors specific for opsonized particles, complement, and several bacterial proteins. Pathogens are recognized by these receptors, which leads to their phagocytosis and subsequent intracellular killing. In addition, the activated macrophages secrete cytokines that affect the function of other cells in the absence of cell contact. Activated macrophages produce large quantities of IL-12 and TNF- $\alpha$, which are cytokines that are responsible for both the recruitment and activation of effector T cells. ${ }^{15}$

\section{2) Dendritic cells}

Dendritic cells (DCs) are phagocytic cells, which, like the macrophages, originate from blood monocytes or a common progenitor in the bone marrow. DCs have a potent ability to initiate adaptive immune responses against pathogens. When intestinal DCs are activated, they migrate to T-cell areas of the gut lymphoid tissue and induce effector T-cell responses. Of importance, intestinal DCs can induce the mucosal receptor $\alpha 4 \beta 7$ and the chemokine receptor CCR9 on T cells. ${ }^{19}$

\section{Atypical lymphocytes}

The atypical lymphocytes and natural killer T cells are also a part of the innate immune response system..$^{20}$ Upon activation, they secrete proinflammatory cytokines and can kill infected or malignant cells. Of note, natural killer T cells producing large amounts of IL-13 have been found in the intestinal epithelium of patients with UC. ${ }^{21}$

\section{Paneth cells, defensins, and the autophagy pathway}

Paneth cells are epithelial cells found at the base of the small intestinal crypts which can sense luminal microbiota and antigens and secrete antimicrobial peptides to contribute to innate immunity. ${ }^{22}$ Paneth cells in the terminal ileum are an abundant source of NOD2 protein; polymorphisms in NOD2, especially in Paneth cells, have been shown to decrease the production of $\alpha$-defensins by these cells. ${ }^{23,24}$

Autophagy is a process that involves degradation and recycling of intracellular contents and removal of intracellular microbes and is mediated by lysosomes. ${ }^{25}$ Genomewide association studies first pointed to the role of autophagy in CD pathogenesis, with many $\mathrm{CD}$-associated genetic loci categorized as having autophagy homeostatic function. ${ }^{26,27}$ Homozygosity for the autophagy risk allele (ATG16L1) contributes to Paneth cell dysfunction in mice and humans, but mice do not have development of spontaneous intestinal inflammation.

\section{TARGETING ACUTE INFLAMMATION}

\section{Cytokines}

In patients with IBD, a large variety of cytokines are derived from different cells of the mucosal immune system, including intestinal epithelial cells, macrophages, natural killer cells, mucosal effector T cells (T helper [Th] 1, 2, and 17), and regulatory $\mathrm{T}$ cells (TRegs). The delicate regulatory mechanisms between proinflammatory and anti-inflammatory cytokines in the mucosa of patients with IBD are yet to be refined. Nevertheless, several approaches to cytokine-based therapy have already been initiated with differing results regarding efficacy and safety. ${ }^{28}$

Of importance is the IL-12 family (IL-12, IL-23, IL-27 and IL$35)$, which involves cytokines produced by antigen-presenting cells during intestinal inflammation. In models of experimental colitis, IL-23 rather than IL-12 has been suggested to be the main determinant of chronic intestinal inflammation. ${ }^{29,30}$

\section{Interferon production}

Antigen-presenting cells can also produce various cytokines of the interferon (IFN) family such as IFN $\alpha$ and IFN $\beta$. Although one study showed that treatment with recombinant IFN $\beta 1$ a was safe, it had no clear positive effect in corticosteroid-refractory UC. ${ }^{31}$ Immunostimulatory techniques to induce IFN production have also been hypothesized to be useful in IBD therapeutics. ${ }^{28}$

\section{3. $T$ cells in IBD}

TNF- $\alpha$ is produced by mucosal T cells, fibroblasts, macro- 
phages, and adipocytes; its blockade represents a successful therapy for CD and UC. When naïve T cells encounter their cognate antigen, they differentiate into effector T cells or TRegs, depending on the cytokine environment at the time. ${ }^{31,32}$ Current research indicates that the balance of different T-cell lineages is altered in IBD and that T-cell signaling and function are altered in $\mathrm{CD}$ and $\mathrm{UC}^{33,34}$

\section{1) Cytokines produced by T effector cells (Th cells)}

Leukocyte infiltration into the intestine is important in IBD initiation and perpetuation. In addition, chemokines and chemokine receptors coordinate trafficking of leukocytes. ${ }^{35}$ Many studies support the concept that activated CD4+ T cells represent the basic immune characteristic of human IBD. In detail, the naïve $\mathrm{T}$ cells can differentiate into three types of effector Th cells: Th1, Th2, and Th17. Of note, CD4+ T cells are activated in the presence of IL-12, and in the absence of IL- 4 they acquire a Th1 phenotype. In addition, CD4+ T cells that are activated in the presence of IL-4 acquire a Th2 phenotype and further lead to the production of IL-4, IL-5, and IL-13. Finally, activation of those cells in the presence of IL-6, transforming growth factor $\beta$ (TGF $\beta$ ), and IL-23 results in expressing the Th17 phenotype. The final phenotype of effector $\mathrm{T}$ cells depends on the antigens and the cytokine milieu present at the time of activation, but details of these phenomena still remain largely unkown. ${ }^{15}$

IL-12 testing is a good example showing a difference between the paths from bench to bedside and that, in some instances, theory that IL-12 could be beneficial to IBD patients contradicts the results of clinical practice showing quite disappointing results with IL-12 use in IBD. In general, the contradictory results between theory and practice concerning interleukin use emphasizes that the concept of a simple Th1/Th2 imbalance in IBD still needs a better investigation. ${ }^{36}$

\section{2) Cytokines produced by TRegs}

TRegs represent a T-cell subset with great immunological importance as molecular targets for IBD therapy. It has been hypothesized that increasing the TReg population/function is of clinical value in IBD therapy.

TRegs are activated by IL-10 and TGF $\beta$, which are mainly produced by DCs. ${ }^{37}$ In addition, TRegs indirectly control the production of TNF- $\alpha$, IL-10, and TGF $\beta$, which are cytokines known to be involved in the regulation of inflammation. IL-10 activates different signaling pathways to exert its anti-inflammatory action, and it has been suggested that IL-10 could represent an important potential therapeutic target in IBD. ${ }^{37}$ However, no clear therapeutic effect of IL-10 has yet been demonstrated in human clinical trials. However, newer formulations of IL-10 targeted at sites of inflammation, such as F8-IL-10 (Dekavil) may show promise for the treatment of IBD. Dekavil is an immunocytokine consisting of a targeting antibody fused to the anti-inflammatory cytokine IL-10. Dekavil has entered Phase II clinical trials in combination with methotrexate for the therapy of Rheumatoid Arthritis and investigations in IBD indications are being considered. Finally, the TGF $\beta 1$ signaling pathway and upregulation of Smad7-the inhibitory molecule of the TGF $\beta 1$ signal-has been of particular interest in preliminary studies. ${ }^{38}$

\section{Multiple cytokine inhibition}

Each of the cytokines above has been discussed as a single entity and as an isolated therapeutic opportunity in the panel of strategies for IBD. Another perspective could be that of targeting multiple cytokines simultaneously to control disease activity. This concept parallels the concept that, in the inflamed mucosa, a variety of cytokines interact together. One example of this multifocused strategy is tofacitinib, a small-molecule Janus-activated kinase (JAK) inhibitor. JAKs are a family of cytoplasmic enzymes that are important to downstream signaling of many cytokines after interacting with the transmembrane receptor. ${ }^{39}$ Another approach is the use of bispecific monoclonal antibodies (BsMAb, BsAb) that may target more than one antiinflammatory cytokines or pathways could be an important therapeutic advancement.

\section{TARGETING SIGNALING AND TRAFFICKING PATHWAYS}

The identification of new mechanisms in IBD pathophysiology has pointed to more specific molecular targets for therapy, including those interfering with inhibition of leukocyte trafficking to the gut. ${ }^{40}$ Upon activation, T cells express several adhesion molecules that direct the $\mathrm{T}$ cells to the sites of inflammation. ${ }^{41}$ In detail, T cells stimulated by mucosal DCs are destined to return to the intestinal epithelium through the upregulation of $\alpha 4 \beta 7$ integrin and CCR9, a chemokine receptor. ${ }^{42}$ The integrin $\alpha 4$ binds to vascular adhesion molecule 1 , which is expressed at sites of inflammation of the vascular endothelium. The $\alpha 4 \beta 7$ integrin recognizes mucosal addressin cellular adhesion molecule-1 (MAdCAM-1), a vascular endothelial molecule, and directs migration of effector $\mathrm{T}$ cells to the intestinal lamina propria. $^{43,44}$

Several other compounds that interfere with gut homing have been evaluated, including antibodies against MAdCAM-1, which was shown to be effective in moderate-severe UC. ${ }^{45}$ Moreover, etrolizumab, an anti- $\beta 7$-antibody, has shown promising results against UC. ${ }^{46} \beta 7$ appears in combination with both $\alpha 4$ and $\alpha \mathrm{E}$; therefore, etrolizumab might have a dual mechanism of action. Information about $\alpha \mathrm{E} \beta 7$ is scarce, but $\alpha 4 \beta 7$ may be downregulated on CD8+ T cells after reaching intestinal lamina propria. ${ }^{47}$ In contrast, $\alpha E \beta 7$ may interact with E-cadherin, its primary ligand, on the basolateral side of the intestinal epithelium; this, then, may promote retention of lymphocytes in the epithelial compartment. ${ }^{36}$ Although many studies have focused on the vascular endothelium and angiogenic mechanisms, studies on the lymphatic vasculature and its functions in IBD are scarce. ${ }^{48}$ 


\section{TARGETING PERPETUATION OF CHRONIC INFLAMMATION}

Initiation and perpetuation of chronic inflammation rely on immunity mechanisms and oxidative stress balance. 0xidative stress has a crucial role in intestinal inflammation. When inflammation is initiated, activated leukocytes produce a wide spectrum of proinflammatory cytokines and also trigger oxidative reactions, which affect the redox equilibrium within the gut mucosa. This oxidative stress-induced imbalance maintains inflammation by initiating redox-sensitive signaling pathways and proinflammatory transcription molecules. ${ }^{49}$ The tremendous progress made in biochemistry and molecular medicine highlights the theoretical possibility of optimal regulation of the oxidative imbalance associated with IBD. For example, IBD could possibly be treated by inducing local production of antiinflammatory molecules, as well as by developing biologic therapies that target selective molecules affecting redox balance and molecular signaling (e.g., inflammasomes). ${ }^{50,51}$

\section{TARGETING MUCOSAL HEALING AND TISSUE DESTRUCTION}

The ultimate goal of histologic healing in IBD is still far from becoming a reality, and the closest therapeutic scenario related to clinical practice is biological healing, which is directly related to mucosal healing..$^{52}$ Mucosal healing depends on the functional harmonization of several types of intestinal cells, including epithelial cells, goblet cells, and Paneth cells. Important steps in mucosal healing include cell proliferation, migration, expansion, and differentiation leading to mucosal healing and intestinal epithelial normalization. ${ }^{20}$ In addition, DCs, adipocytes, fibroblasts, myoblasts have an important role in mucosal healing and regeneration (Table 1). These cells promote the healing and regeneration process through a very well balanced function which is regulated by special molecular mediators and tissue repair feedback signaling.

Although it is difficult to discern which factors are of importance in determining these events and which are bystanders in these mucosal healing phenomena, it would be important to delineate factors that could be critical in the process of mucosal healing. This approach of revealing the optimal strategies for mucosal healing could also point toward new therapeutic molecules for IBD.

\section{CRITICAL OVERVIEW OF MOLECULAR TARGETS IN IBD THERAPY}

IBD therapy has advanced considerably in the past 2 decades with the use of biologic agents, mostly anti-TNF- $\alpha$-based therapies. However, it is clear that the therapeutic needs in IBD are still unmet, and the efficacy of these new therapies is limited in some groups of patients. Several novel strategies have been developed to address these needs of IBD patients for more ef- fective and safer therapies. These strategies include reinforcing epithelial barrier function, proinflammatory cytokine inhibition, blocking of inflammatory cell trafficking, and enhancing TReg function (Table 2)..$^{53}$

Advances in molecular genetics have shown that several IBD-related genes involved in immune pathways could represent useful therapeutic targets. ${ }^{54}$ In addition, dysregulation of crosstalk among several molecular pathways in IBD still needs further elucidation. An example of the dynamic interaction of adaptive immunity is in the role of Th17 cells in IBD. Patients with CD have been shown to have increased Th17 cells; hence, anti-IL-17 antibody (secukinumab) was tried as a therapeutic strategy. Unexpectedly, secukinumab resulted in the worsening of $\mathrm{CD}$ and more adverse events in treated patients, which was attributed to the complex biology of Th17 cells.

A drawback of current clinical trials is inadequate or lack of immunophenotyping of patients. However, recent advances in high-throughput technologies provide an opportunity to monitor the dynamics and the complexity of the immune system, which may to lead to a more personalized treatment approach in IBD. ${ }^{15}$ However, there are many unanswered questions about the best therapies, the long-term safety of biologic agents, and the ability of new therapies to change the natural history of IBD. $^{55}$

It is important to mention that different drugs could produce similar therapeutic effects regarding modification of inflammatory pathways. Many studies ${ }^{40-44}$ have challenged new perspectives on the discovery of molecular targets for definitive IBD therapy. We have learned that experimental data do not always correspond to disease in clinical settings and that many improvements and refinements of our molecular approach to IBD have yet to be done. New studies assessing the complex immune signatures in response to different therapies are needed. ${ }^{56}$

After the breakthrough of TNF- $\alpha$ blockade by neutralizing antibodies in both CD and UC, many therapeutic agents inhibiting the activity of proinflammatory cytokines or supporting the action of anti-inflammatory cytokines were evaluated as therapy for IBD. ${ }^{39,40}$ Unfortunately, many of them failed in clinical studies or had beneficial effects in subgroups of patients only, which underscores the notion that cytokine networks in human IBD are more complex than previously assumed and may vary among patients. This poses the question of whether further and profound progress can be made with targeting cytokines in IBD.

A possible answer is to target several cytokines at the same time, through a combination of different targeted antibodies, through interfering with overlapping intracellular signaling pathways, or through enhancement of anti-inflammatory cytokines. However, it does not seem wise to randomly test possible combinations. Instead, it is mandatory that we increase and repeatedly test our knowledge about the pathogenesis of IBD using model systems. ${ }^{57}$ As an example, the clinical phenotype may be similar between two patients with IBD, but the underlying 
Table 2. Critical Overview and Future Directions of Molecular Targets in Inflammatory Bowel Disease Therapy

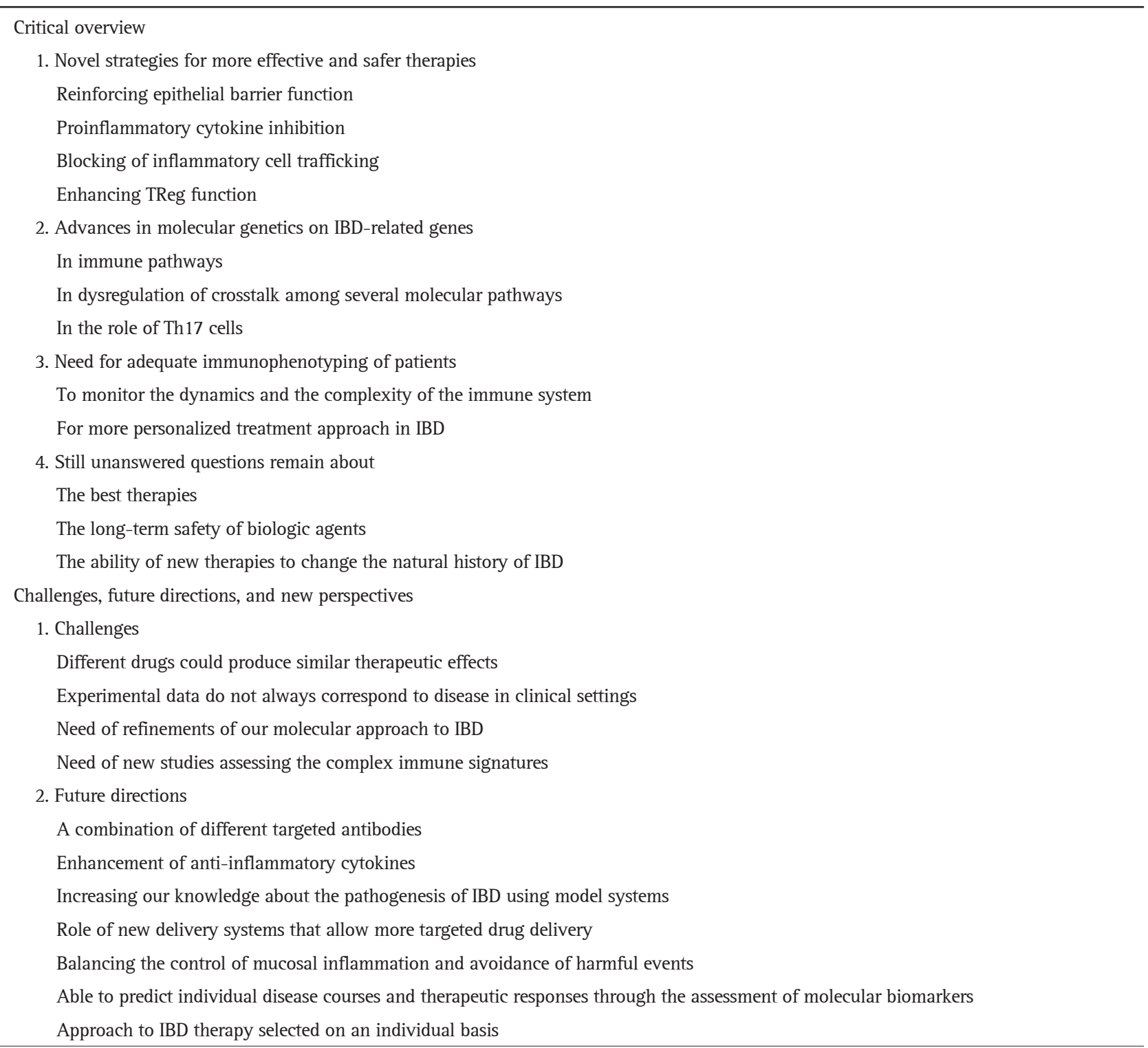

TReg, regulatory T cell; IBD, inflammatory bowel disease; Th, T helper.

aberrations in the immune system could be very different. Even in the same patient, the underlying immunopathologic processes may change over time, regardless of the initial triggering events.

Finally, the expanding role of new delivery systems that allow more targeted drug delivery is also expected to benefit IBD therapeutics.

These new delivery systems that allow more targeted drug delivery include for example nanomedicine which represents a major challenging field with its main aims: targeted drug delivery, diagnostic, theranostics, tissue engineering, and personalized medicine. Although the real applications of these systems still need major work, nevertheless new challenges are open, and perspectives based on integrated multidisciplinary approaches would enable both a deeper basic knowledge and the expected advances in biomedical field.

The great challenge of balancing the control of mucosal inflammation and avoidance of harmful events remains to be successfully addressed. ${ }^{58}$

\section{CONCLUSIONS}

Despite the increased knowledge gained from animal and human studies, many aspects of mucosal immunity in patients with IBD remain unclear. Recently, significant progress has been made in high-throughput technologies such as genomic sequencing, which provide multiparametric data that can be used to not only define the various immune cell states but also assess how they interact with each other in various conditions. 
More detailed knowledge of the complexity of the immune system in IBD will improve the classification of IBD, the development of more representative animal models, and the design of new biologic therapies for a more personalized treatment approach. ${ }^{59}$ Once we are sufficiently able to predict individual disease courses and therapeutic responses through the assessment of molecular biomarkers, it seems that the most encouraging approach to IBD therapy will be selected on an individual basis.

\section{CONFLICTS OF INTEREST}

No potential conflict of interest relevant to this article was reported.

\section{REFERENCES}

1. Holtmann MH, Neurath MF. From immunogenic mechanisms to novel therapeutic approaches in inflammatory bowel disease. Adv Exp Med Biol 2006;579:227-242.

2. Molodecky NA, Soon IS, Rabi DM, et al. Increasing incidence and prevalence of the inflammatory bowel diseases with time, based on systematic review. Gastroenterology 2012;142:46-54.e42.

3. Gabbani T, Deiana S, Annese AL, Lunardi S, Annese V. The genetic burden of inflammatory bowel diseases: implications for the clinic? Expert Rev Gastroenterol Hepatol 2016;10:1109-1117.

4. Vogelaar L, Spijker AV, van der Woude CJ. The impact of biologics on health-related quality of life in patients with inflammatory bowel disease. Clin Exp Gastroenterol 2009;2:101-109.

5. Bernstein CN, Loftus EV Jr, Ng SC, Lakatos PL, Moum B; Epidemiology and Natural History Task Force of the International Organization for the Study of Inflammatory Bowel Disease (IOIBD). Hospitalisations and surgery in Crohn's disease. Gut 2012;61:622629.

6. Barker N, van Es JH, Kuipers J, et al. Identification of stem cells in small intestine and colon by marker gene Lgr5. Nature 2007;449:10031007.

7. Fasano A, Shea-Donohue T. Mechanisms of disease: the role of intestinal barrier function in the pathogenesis of gastrointestinal autoimmune diseases. Nat Clin Pract Gastroenterol Hepatol 2005;2:416-422.

8. Khor B, Gardet A, Xavier RJ. Genetics and pathogenesis of inflammatory bowel disease. Nature 2011;474:307-317.

9. Prager M, Büttner J, Haas V, et al. The JAK2 variant rs10758669 in Crohn's disease: altering the intestinal barrier as one mechanism of action. Int J Colorectal Dis 2012;27:565-573.

10. Stoll M, Corneliussen B, Costello CM, et al. Genetic variation in DLG5 is associated with inflammatory bowel disease. Nat Genet 2004;36:476-480.

11. Atreya R, Neurath MF. IBD pathogenesis in 2014: molecular pathways controlling barrier function in IBD. Nat Rev Gastroenterol Hepatol 2015;12:67-68.

12. Günther C, Buchen B, Neurath MF, Becker C. Regulation and pathophysiological role of epithelial turnover in the gut. Semin Cell Dev Biol 2014;35:40-50.

13. Becker C, Watson AJ, Neurath MF. Complex roles of caspases in the pathogenesis of inflammatory bowel disease. Gastroenterology 2013;144:283-293.

14. Coskun M. The role of CDX2 in inflammatory bowel disease. Dan Med J 2014;61:B4820.

15. Dave M, Papadakis KA, Faubion WA Jr. Immunology of inflammatory bowel disease and molecular targets for biologics. Gastroenterol Clin North Am 2014;43:405-424.

16. Cristofaro P, Opal SM. Role of Toll-like receptors in infection and immunity: clinical implications. Drugs 2006;66:15-29.

17. Li J, Wang X, Zhang F, Yin H. Toll-like receptors as therapeutic targets for autoimmune connective tissue diseases. Pharmacol Ther 2013;138:441-451.

18. Pochini L, Scalise M, Galluccio M, Indiveri C. OCTN cation transporters in health and disease: role as drug targets and assay development. J Biomol Screen 2013;18:851-867.

19. Ayabe T, Satchell DP, Wilson CL, Parks WC, Selsted ME, Ouellette AJ. Secretion of microbicidal alpha-defensins by intestinal Paneth cells in response to bacteria. Nat Immunol 2000;1:113-118.

20. Neurath MF. New targets for mucosal healing and therapy in inflammatory bowel diseases. Mucosal Immunol 2014;7:6-19.

21. Fuss IJ, Heller F, Boirivant M, et al. Nonclassical CD1d-restricted NK T cells that produce IL-13 characterize an atypical Th2 response in ulcerative colitis. J Clin Invest 2004;113:1490-1497.

22. Vaishnava S, Behrendt CL, Ismail AS, Eckmann L, Hooper LV. Paneth cells directly sense gut commensals and maintain homeostasis at the intestinal host-microbial interface. Proc Natl Acad Sci U S A 2008;105:20858-20863.

23. Wehkamp J, Harder J, Weichenthal M, et al. NOD2 (CARD15) mutations in Crohn's disease are associated with diminished mucosal alpha-defensin expression. Gut 2004;53:1658-1664.

24. Lala S, Ogura Y, Osborne C, et al. Crohn's disease and the NOD2 gene: a role for paneth cells. Gastroenterology 2003;125:47-57.

25. Boya P, Reggiori F, Codogno P. Emerging regulation and functions of autophagy. Nat Cell Biol 2013;15:713-720.

26. Rioux JD, Xavier RJ, Taylor KD, et al. Genome-wide association study identifies new susceptibility loci for Crohn disease and implicates autophagy in disease pathogenesis. Nat Genet 2007;39:596-604.

27. McCarroll SA, Huett A, Kuballa P, et al. Deletion polymorphism upstream of IRGM associated with altered IRGM expression and Crohn's disease. Nat Genet 2008;40:1107-1112.

28. Neurath MF. Cytokines in inflammatory bowel disease. Nat Rev Immunol 2014;14:329-342.

29. Cordero Ruiz P, Castro Márquez C, Méndez Rufián V, et al. Efficacy of adalimumab in patients with Crohn's disease and failure to infliximab therapy: a clinical series. Rev Esp Enferm Dig 2011;103:294-298

30. Corleto VD, Pagnini C, Margagnoni G, et al. IL-1beta-511 and IL$1 \mathrm{RN}^{*} 2$ polymorphisms in inflammatory bowel disease: an Italian 
population study and meta-analysis of European studies. Dig Liver Dis 2010;42:179-184.

31. Atreya R, Zimmer M, Bartsch B, et al. Antibodies against tumor necrosis factor (TNF) induce T-cell apoptosis in patients with inflammatory bowel diseases via TNF receptor 2 and intestinal CD14 ${ }^{+}$macrophages. Gastroenterology 2011;141:2026-2038.

32. Kamada N, Hisamatsu T, Okamoto S, et al. Unique CD14 intestinal macrophages contribute to the pathogenesis of Crohn disease via IL-23/IFN-gamma axis. J Clin Invest 2008;118:2269-2280.

33. Zenewicz LA, Antov A, Flavell RA. CD4 T-cell differentiation and inflammatory bowel disease. Trends Mol Med 2009;15:199-207.

34. Isaacs KL, Lewis JD, Sandborn WJ, Sands BE, Targan SR. State of the art: IBD therapy and clinical trials in IBD. Inflamm Bowel Dis 2005;11 Suppl 1:S3-S12.

35. Nishimura M, Kuboi Y, Muramoto K, Kawano T, Imai T. Chemokines as novel therapeutic targets for inflammatory bowel disease. Ann N Y Acad Sci 2009;1173:350-356.

36. Zundler S, Neurath MF. Immunopathogenesis of inflammatory bowel diseases: functional role of $\mathrm{T}$ cells and $\mathrm{T}$ cell homing. Clin Exp Rheumatol 2015;33(4 Suppl 92):S19-S28.

37. Coombes JL, Siddiqui KR, Arancibia-Cárcamo CV, et al. A functionally specialized population of mucosal CD103+ DCs induces Foxp3+ regulatory T cells via a TGF-beta and retinoic acid-dependent mechanism. J Exp Med 2007;204:1757-1764.

38. Kelsall B. Interleukin-10 in inflammatory bowel disease. N Engl J Med 2009;361:2091-2093.

39. McLean MH, Neurath MF, Durum SK. Targeting interleukins for the treatment of inflammatory bowel disease-what lies beyond anti-TNF therapy? Inflamm Bowel Dis 2014;20:389-397.

40. Mosli MH, Rivera-Nieves J, Feagan BG. T-cell trafficking and antiadhesion strategies in inflammatory bowel disease: current and future prospects. Drugs 2014;74:297-311.

41. Johansson-Lindbom B, Svensson M, Pabst 0, et al. Functional specialization of gut CD103+ dendritic cells in the regulation of tissue-selective T cell homing. J Exp Med 2005;202:1063-1073.

42. Mora JR, Bono MR, Manjunath N, et al. Selective imprinting of gut-homing $\mathrm{T}$ cells by Peyer's patch dendritic cells. Nature 2003;424:88-93.

43. Berlin C, Berg EL, Briskin MJ, et al. Alpha 4 beta 7 integrin mediates lymphocyte binding to the mucosal vascular addressin MAdCAM-1. Cell 1993;74:185-195.

44. Briskin M, Winsor-Hines D, Shyjan A, et al. Human mucosal addressin cell adhesion molecule-1 is preferentially expressed in intestinal tract and associated lymphoid tissue. Am J Pathol 1997;151:97-110.

45. Vermeire S, Sandborn W, Danese S et al. A randomized, multicenter double-blind, placebo-controlled study of the safety and efficacy of anti-MAdCAM antibody PF-00547659 (PF) in patients with moderate to severe ulcerative colitis (UC): results of the TURANDOT study. J Crohns Colitis 2015;148(4 Suppl 1):S1193.

46. Vermeire S, O’Byrne S, Keir M, et al. Etrolizumab as induction therapy for ulcerative colitis: a randomised, controlled, phase 2 trial. Lancet 2014;384:309-318.

47. Ericsson A, Svensson M, Arya A, Agace WW. CCL25/CCR9 promotes the induction and function of CD103 on intestinal intraepithelial lymphocytes. Eur J Immunol 2004;34:2720-2729.

48. D'Alessio S, Tacconi C, Fiocchi C, Danese S. Advances in therapeutic interventions targeting the vascular and lymphatic endothelium in inflammatory bowel disease. Curr Opin Gastroenterol 2013;29:608-613.

49. Biasi F, Leonarduzzi G, Oteiza PI, Poli G. Inflammatory bowel disease: mechanisms, redox considerations, and therapeutic targets. Antioxid Redox Signal 2013;19:1711-1747.

50. Tokuhira N, Kitagishi Y, Suzuki M, et al. PI3K/AKT/PTEN pathway as a target for Crohn's disease therapy (Review). Int J Mol Med 2015;35:10-16.

51. Schottelius AJ, Baldwin AS Jr. A role for transcription factor NF-kappa B in intestinal inflammation. Int J Colorectal Dis 1999;14:18-28.

52. Scaldaferri F, Petito V, Lopetuso L, et al. Pre- and posttherapy assessment of intestinal soluble mediators in IBD: where we stand and future perspectives. Mediators Inflamm 2013;2013:391473.

53. Malik T, Mannon P. Inflammatory bowel diseases: emerging therapies and promising molecular targets. Front Biosci (Schol Ed) 2012;4:1172-1189.

54. Leppkes M, Neurath MF, Herrmann M, Becker C. Immune deficiency vs. immune excess in inflammatory bowel diseasesSTAT3 as a rheo-STAT of intestinal homeostasis. J Leukoc Biol 2016;99:57-66.

55. Panaccione R, Ferraz JG, Beck P. Advances in medical therapy of inflammatory bowel disease. Curr Opin Pharmacol 2005;5:566572.

56. Cole AT, Hawkey CJ. Treatment of inflammatory bowel disease from now to the millennium. Baillieres Clin Gastroenterol 1994;8:351-379.

57. Zundler S, Neurath MF. How will new and future therapies change our treatment of IBD? Expert Rev Clin Immunol 2016;12:233-236.

58. Leiman DA, Lichtenstein GR. Therapy of inflammatory bowel disease: what to expect in the next decade. Curr Opin Gastroenterol 2014;30:385-390.

59. Bene L, Falus A, Baffy N, Fulop AK. Cellular and molecular mechanisms in the two major forms of inflammatory bowel disease. Pathol Oncol Res 2011;17:463-472. 